\title{
Atuação da enfermagem no serviço de radioterapia (1998-2018)
}

\section{Performance of nursing in the radiotherapy service (1998-2018)}

DOI: $10.46919 / \operatorname{archv1n5-001}$

Recebimento dos originais: 10/07/2020

Aceitação para publicação: 30/08/2020

\section{Mário Sérgio Bruggmann}

Enfermeiro. Doutorando em Enfermagem.

Instituição: Programa de Pós-Graduação em Enfermagem da Universidade Federal de Santa Catarina (UFSC)

Endereço: Rua Delfino Conti, Trindade, Florianópolis, Santa Catarina - Brasil

E-mail: mariobrugg@gmail.com

\section{Carolina Neis Machado}

Fisioterapeuta. Doutoranda em Enfermagem.

Instituição: Programa de Pós-Graduação em Enfermagem da Universidade Federal de Santa Catarina (UFSC)

Endereço: Rua Delfino Conti, Trindade, Florianópolis, Santa Catarina - Brasil

E-mail: carolina.neis@ posgrad.ufsc.br

\section{Maiara Suelen Mazera}

Enfermeira. Doutoranda em Enfermagem

Instituição: Programa de Pós-Graduação em Enfermagem da Universidade Federal de Santa Catarina (UFSC)

Endereço: Rua Delfino Conti, Trindade, Florianópolis, Santa Catarina - Brasil

E-mail: maiaramazera@gmail.com

\section{Dulcinéia Ghizoni Schneider}

Enfermeira. Doutora em Enfermagem. Docente do Programa de Pós-Graduação em Enfermagem da UFSC

Instituição: Programa de Pós-Graduação em Enfermagem da Universidade Federal de Santa Catarina (UFSC)

Endereço: Rua Delfino Conti, Trindade, Florianópolis, Santa Catarina - Brasil

E-mail: dulcineiags@gmail.com

\section{Denise Elvira Pires de Pires}

Enfermeira. Doutora em Enfermagem. Docente do Programa de Pós-Graduação em Enfermagem da UFSC

Instituição: Programa de Pós-Graduação em Enfermagem da Universidade Federal de Santa Catarina (UFSC)

Endereço: Rua Delfino Conti, Trindade, Florianópolis, Santa Catarina - Brasil

E-mail: piresdp@yahoo.com 


\title{
RESUMO
}

Objetivo: analisar a produção científica sobre a atuação da enfermagem no serviço de radioterapia no período de 1998 a 2018. Método: revisão integrativa da literatura realizada nas bases de dados Literatura Latino-Americano e do Caribe em Ciências da Saúde, Web of Science e Medical Literature and Retrivial System Online, em maio de 2018. A amostra foi composta por 15 publicações. Resultados: o trabalho da enfermagem no serviço de radioterapia está relacionado ao processo de enfermagem, consulta de enfermagem, cuidado humanizado, abordagem de cuidado centrado na pessoa (person-centred care), educação permanente em saúde e efeitos adversos da radioterapia (lesões de pele e mucosa). Conclusão: o trabalho da enfermagem no serviço de radioterapia é estruturado a partir do Processo de Enfermagem, desenvolvido mediante as necessidades biopsicossociais da pessoa submetida à radioterapia. $\mathrm{O}$ estudo fornece subsídios para reflexão e reconhecimento sobre o trabalho desenvolvido pela enfermagem no serviço de radioterapia.

Palavras-chave: Enfermagem, Radioterapia, Serviços de enfermagem, Cuidados de enfermagem, Educação em Enfermagem.

\begin{abstract}
Objective: to analyze the scientific production on the performance of nursing in the radiotherapy service from 1998 to 2018. Method: integrative literature review carried out in the databases of Latin American and Caribbean Literature in Health Sciences, Web of Science and Medical Literature and Retrivial System Online, in May 2018. The sample consisted of 15 publications. Results: the nursing work in the radiotherapy service is related to the nursing process, nursing consultation, humanized care, personcentered care approach, permanent health education and adverse effects of radiotherapy (skin lesions and mucosa). Conclusion: the nursing work in the radiotherapy service is structured based on the Nursing Process, developed through the biopsychosocial needs of the person submitted to radiotherapy. The study provides subsidies for reflection and recognition about the work developed by nursing in the radiotherapy service.
\end{abstract}

Keywords: Nursing, Radiotherapy, Nursing Services, Nursing Care, Education, Nursing.

\section{INTRODUÇÃO}

Atualmente, as doenças oncológicas possuem expressividade no cenário das doenças crônicas não transmissíveis. Para substanciar este fato, a Organização Mundial da Saúde (OMS) estima 21,4 milhões de casos incidentes para o ano de 2030, em decorrência do crescimento e do envelhecimento da população. ${ }^{1}$ No Brasil, dados estatísticos direcionam para uma ocorrência equivalente ao aparecimento de cerca de 580 mil novos casos de câncer, revelando, desta forma, a magnitude do problema no país. ${ }^{2}$

A radioterapia é um importante recurso terapêutico aplicado às neoplasias. Constitui-se como um tratamento localizado, que usa radiação ionizante produzida por aparelhos ou emitida por radioisótopos naturais. É, na sua grande maioria, feita em regime ambulatorial ${ }^{3}$ e envolve várias etapas e profissionais de saúde, a exemplo de enfermeiros, médicos, físicos, técnicos e tecnólogos em radiologia, além do uso de elaborados hardwares e softwares. ${ }^{4-6}$ A finalidade do tratamento é entregar ao tecido alvo do paciente, uma quantidade de dose de radiação ionizante efetiva e segura. ${ }^{7}$ 
Pacientes que necessitam de radioterapia enfrentam uma série de desafios para seu bem-estar. O tipo de tumor, os fatores de tratamento e as características pessoais farão com que cada um reaja de modo diferente a essa abordagem terapêutica. ${ }^{8}$ Apesar de ser um tratamento eficaz, pode ocasionar diversas manifestações clínicas, agudas e crônicas, conhecidas como efeitos adversos. As reações de pele (radiodermites, eritemas), náuseas, mucosite, xerostomia, fadiga, anorexia, diarreia e disfagia estão entre os principais. ${ }^{9}$ A prevenção e o controle desses efeitos são de fundamental importância, pois podem limitar o tratamento, levar a sua interrupção temporária ou definitiva, diminuir a motivação do paciente em prosseguir e, dessa forma, comprometer o controle local do tumor e as taxas de sobrevida. ${ }^{10}$

O acompanhamento dos pacientes em tratamento radioterapêutico ocorre no contexto de uma equipe multidisciplinar, mas, frequentemente, são os profissionais de enfermagem que estabelecem o contato inicial, além de serem aqueles que fazem os encaminhamentos a outros profissionais da saúde, quando necessário. ${ }^{8}$

A inserção da enfermagem no serviço de radioterapia se deu com a implantação do Projeto Expande,${ }^{11}$ do Ministério da Saúde, tendo como finalidade a garantia de atendimento integral às pessoas com doenças neoplásicas malignas, em todo o país. A Resolução No 211/1998 ${ }^{12}$ do Conselho Federal de Enfermagem (COFEN), que dispõe sobre a atuação dos profissionais de enfermagem que trabalham com radiação ionizante em Radioterapia, Medicina Nuclear e Serviços de Imagem foi elaborada, considerando a necessidade de regulamentar o trabalho realizado pela enfermagem nestes ambientes e assegurar condições adequadas de trabalho a estes profissionais. Tal resolução assinala ainda, que o enfermeiro deve estar inserido nas atividades gerenciais, assistenciais e de caráter educativo ao paciente e seus familiares.

Mais recentemente, a Resolução $N^{\circ} 389 / 2011^{13}$ do COFEN regulamentou que a assistência à pessoa com doença oncológica deve ser prestada por enfermeiro especializado, que tenha conhecimento científico aprofundado e habilidades técnicas para a resolução de situações clínicas de maior complexidade. Além disso, estabelece que este profissional deve promover uma relação de confiança com o paciente, oferecendo uma assistência de qualidade e humanizada, com o intuito de reduzir os efeitos do tratamento.

O planejamento da assistência de enfermagem em radioterapia segue as etapas do método científico, sustentado pela Resolução No 358 de 2009 do Conselho Federal de Enfermagem, que dispõe sobre a Sistematização da Assistência de Enfermagem (SAE) e Processo de Enfermagem (PE). ${ }^{14-15}$

Neste entendimento, é relevante assinalar que os enfermeiros participam ativamente na assistência dos pacientes em tratamento radioterapêutico, sendo que suas funções primordiais integram a orientação sobre os objetivos do tratamento e o planejamento de um cuidado integral, face às necessidades individuais da pessoa e seus familiares. Assim, ao assistir os pacientes submetidos à radioterapia, a equipe 
de enfermagem pode prevenir complicações e/ou minimizar os efeitos adversos evitáveis da terapêutica empregada e perceber os prejuízos emocionais das pessoas envolvidas no tratamento, promovendo uma assistência humanizada. ${ }^{16}$

Os profissionais da área de enfermagem oncológica realizam atividades de grande importância nas consultas, como avaliar as respostas do paciente ao tratamento estabelecido, os efeitos adversos tardios, além das recidivas do câncer. ${ }^{17}$ Atuam em conjunto com médicos radio-oncologistas, com o objetivo de fornecer cuidado de alta qualidade aos pacientes. ${ }^{8}$ Neste sentido, a compreensão do papel do enfermeiro nas diversas áreas de atuação, possibilita o reconhecimento do seu processo de trabalho e confirma a sua importância para a qualidade dos serviços. ${ }^{15}$ É nessa perspectiva que este trabalho de revisão se estabelece.

Com base na importância histórica do trabalho da equipe de enfermagem no serviço de radioterapia, estabelecido a partir das regulamentações supramencionadas, definiu-se a seguinte pergunta de pesquisa: como se dá a atuação da enfermagem no serviço de radioterapia, segundo o encontrado na literatura, no período 1998 a 2018? Este estudo tem como objetivo analisar a produção científica sobre a atuação da enfermagem no serviço de radioterapia no período de 1998 a 2018.

\section{MÉTODO}

Trata-se de uma revisão integrativa da literatura. ${ }^{18}$ Este método de pesquisa permite a síntese de múltiplos estudos publicados e possibilita conclusões gerais a respeito de uma área particular de estudo. ${ }^{19}$ A revisão integrativa da literatura determina critérios precisos sobre o processo investigativo de coleta de dados, análise e apresentação de resultados, desde o início do estudo, mediante um protocolo de pesquisa previamente elaborado e devidamente validado. ${ }^{20}$

A escolha do recorte histórico justifica-se pela Resolução n. 211/1998 ${ }^{21}$ do COFEN, que estabelece a atuação dos profissionais de enfermagem nos procedimentos que envolvem radiação ionizante. A coleta de dados foi realizada em maio de 2018 por meio do Portal de periódicos CAPES. Foram escolhidas as bases de dados Literatura Latino-Americano e do Caribe em Ciências da Saúde (LILACS), Web of Science e Medical Literature and Retrivial System Online (MEDLINE). A escolha dessas fontes aconteceu pela intencionalidade dos pesquisadores em apontar a produção científica sobre a temática nos cenários nacional e internacional. Os descritores utilizados foram: (“enferm\$” OR "nurs\$”) AND ("radiation therapy" OR "radiotherapy" OR "radioterapia”) AND (work OR "work process" OR "processo de trabalho"). 
Fluxograma 1 - Fluxograma baseado no modelo PRISMA para a seleção dos artigos. Florianópolis, 2020.

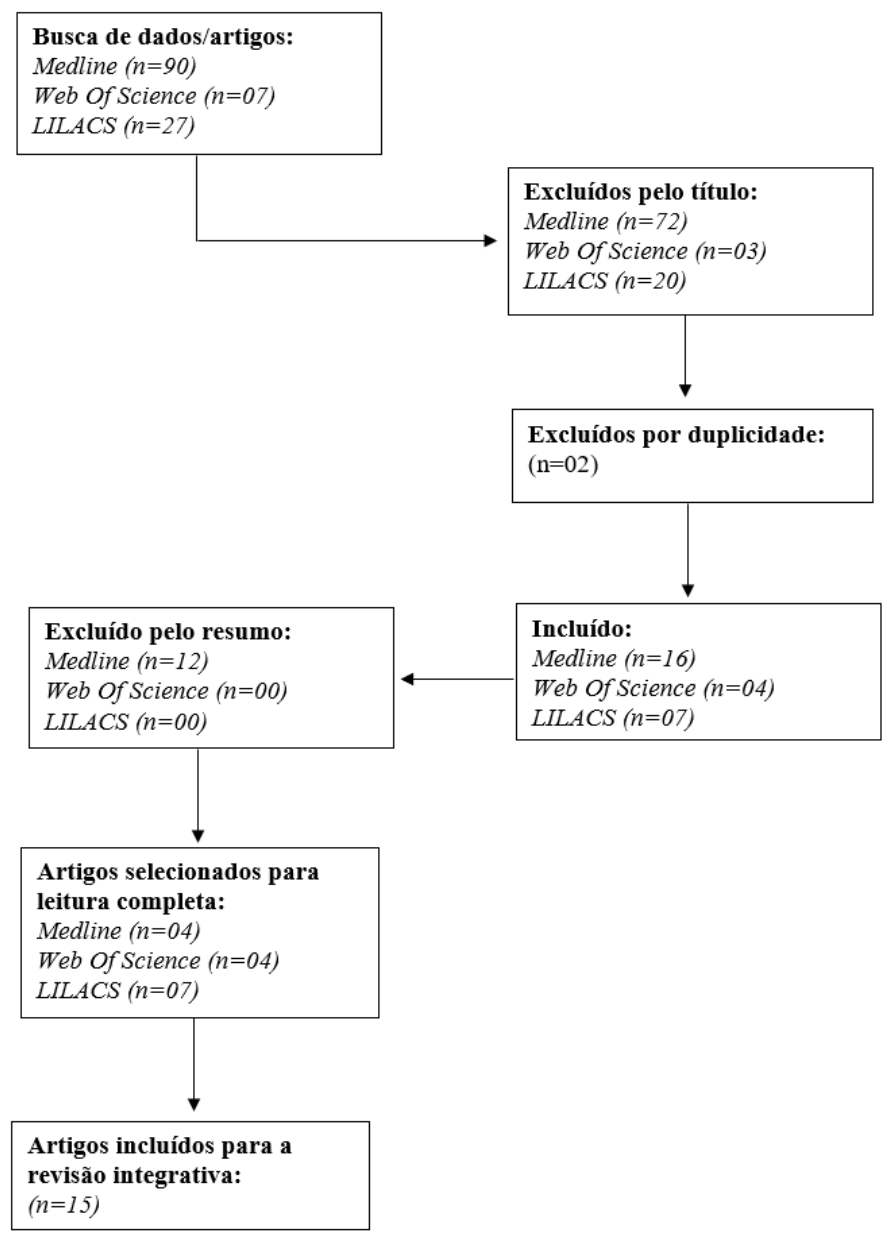

Para a seleção dos artigos, foram adotados os seguintes critérios de inclusão: artigos originais, publicados em inglês ou português, no período de 1998 a 2018. Utilizou-se o critério de qualidade duploindependente na seleção e na extração desses. Os artigos deveriam apresentar descritores no resumo e/ou no título. Nesta fase, foram realizadas leituras dos resumos de cada artigo. Foram excluídas teses, capítulos de teses, livros, capítulo de livros, anais de congressos ou conferências, relatórios técnicos e científicos e documentos ministeriais. Os artigos que se replicavam em mais de uma base de dados foram considerados em apenas uma e automaticamente excluído da outra.

Para a fase de análise e interpretação dos dados, foi construída uma tabela no Programa Microsoft Excel $^{\circledR}$ com as seguintes colunas: base, título do estudo, autores, periódico, ano de publicação e considerações / temática.

Os artigos foram analisados criticamente por meio de leitura na íntegra, para que os autores pudessem se aprofundar na temática descrita e compreender sua relação com o objetivo do estudo. Por fim, foi realizada uma síntese dos resultados dos estudos selecionados, os quais foram organizados em categorias, observando as confluências e divergências dos achados em relação à proposta deste estudo. A 
organização das categorias foi realizada com base na atuação da equipe de enfermagem no serviço de radioterapia, incluindo o Processo de Enfermagem, Educação em Saúde, Humanização na Assistência e Atuação dos Enfermeiros nos casos de Lesões de Pele proveniente da radiação.

\section{RESULTADOS}

Dos 33 artigos selecionados, após a leitura dos resumos, 16 estavam indexados na base de dados MEDLINE, oito na LILACS e quatro na WEB OF SCIENCE. No que se refere aos 15 artigos incluídos no estudo, após leitura integral, estes foram publicados em 13 periódicos diferentes, com destaque para os seguintes: Revista de Enfermagem UERJ e Revista Brasileira de Enfermagem. Em relação ao ano de publicação dos artigos selecionados, a maior concentração foi entre o período de 2009 e 2011, com seis artigos.

O quadro 2 sintetiza as características dos artigos selecionados quanto à base de dados, título, ano de publicação e periódico.

Quadro 1 - Artigos selecionados para a revisão. Florianópolis, 2018.

\begin{tabular}{|c|c|c|c|}
\hline Base & Título do artigo & Ano & Periódico \\
\hline MEDLINE & $\begin{array}{l}\text { Non-physician practitioners in radiation } \\
\text { oncology: advanced practice nurses and } \\
\text { physician assistants }{ }^{17}\end{array}$ & 1999 & $\begin{array}{l}\text { International Journal of Radiation } \\
\text { Oncology }\end{array}$ \\
\hline $\begin{array}{l}\text { WEB OF } \\
\text { SCIENCE }\end{array}$ & $\begin{array}{l}\text { Evaluation of nurse-led follow up for patients } \\
\text { undergoing pelvic radiotherapy }{ }^{22}\end{array}$ & 2001 & British Journal of Cancer \\
\hline LILACS & $\begin{array}{l}\text { Assistência humanizada ao cliente oncológico: } \\
\text { reflexões junto à equipe }{ }^{23}\end{array}$ & 2003 & Revista Brasileira de Enferma \\
\hline MEDLINE & $\begin{array}{l}\text { Specialist breast care nurses for supportive care } \\
\text { of women with breast câncer }{ }^{24}\end{array}$ & 2008 & $\begin{array}{l}\text { The Cochrane } \\
\text { Systematic Reviews }\end{array}$ \\
\hline LILACS & $\begin{array}{l}\text { As teias da sobrevivência oncológica: com a vida } \\
\text { de } \text { novo }^{25}\end{array}$ & 2009 & $\begin{array}{l}\text { Revista Texto \& Contexto } \\
\text { Enfermagem }\end{array}$ \\
\hline LILACS & $\begin{array}{l}\text { Tecnologias emissoras de radiação ionizante e a } \\
\text { necessidade de educação permanente para uma } \\
\text { práxis segura da enfermagem radiológica }{ }^{26}\end{array}$ & 2009 & Revista Brasileira de Enferı \\
\hline LILACS & Prevenção e tratamento da mucosite oral ${ }^{27}$ & 2010 & Revista Enfermagem UERJ \\
\hline LILACS & $\begin{array}{l}\text { Mucosite em pacientes portadores de câncer de } \\
\text { cabeça e pescoço submetidos à } \\
\text { radioquimioterapia }^{28}\end{array}$ & 2011 & $\begin{array}{l}\text { Revista da Escola Enfermagem da } \\
\text { USP }\end{array}$ \\
\hline $\begin{array}{l}\text { WEB OF } \\
\text { SCIENCE }\end{array}$ & $\begin{array}{l}\text { Early diagnosis in primary oral cancer: is it } \\
\text { possible } ?^{29}\end{array}$ & 2011 & $\begin{array}{l}\text { Medicina Oral, Patologia Oral y } \\
\text { Cirurgia Bucal }\end{array}$ \\
\hline MEDLINE & $\begin{array}{l}\text { Person centred nursing care in radiation } \\
\text { oncology: a case study }{ }^{30}\end{array}$ & 2013 & $\begin{array}{l}\text { European Journal } \\
\text { Nursing }\end{array}$ \\
\hline LILACS & $\begin{array}{l}\text { Interdisciplinaridade e integralidade: a } \\
\text { abordagem do assistente social e do enfermeiro } \\
\text { no INCA }\end{array}$ & 2014 & Revista Brasileira de Cancerologia \\
\hline MEDLINE & Profile and predictors of global distress: can the & 2014 & Palliative \& Supportive Care \\
\hline
\end{tabular}




\begin{tabular}{|c|c|c|c|}
\hline & DT guide nursing practice in prostate cancer? $?^{32}$ & & \\
\hline $\begin{array}{l}\text { WEB OF } \\
\text { SCIENCE }\end{array}$ & $\begin{array}{l}\text { Nurse-led group consultation intervention } \\
\text { reduces depressive symptoms in men with } \\
\text { localised prostate cancer: a cluster randomised } \\
\text { controlled trial }^{33}\end{array}$ & 2016 & BMC Cancer \\
\hline LILACS & \begin{tabular}{|l} 
Atuação de enfermeiros em serviços de \\
radioterapia $^{15}$
\end{tabular} & 2017 & Revista Enfermagem UERJ \\
\hline $\begin{array}{l}\text { WEB OF } \\
\text { SCIENCE }\end{array}$ & $\begin{array}{l}\text { Survey of Difficult Experiences of Nurses Caring } \\
\text { for Patients Undergoing Radiation Therapy: An } \\
\text { Analysis of Factors in Difficult Cases }{ }^{34}\end{array}$ & 2018 & $\begin{array}{l}\text { Asia-pacific Journal } \\
\text { Oncology nursing }\end{array}$ \\
\hline
\end{tabular}

As publicações da base MEDLINE abordaram de modo expresivo a assistência da enfermagem como fator preponderante para a qualidade do atendimento ao paciente em radioterapia e, também, para o estabelecimento da abordagem de cuidado centrado na pessoa (person-centred care). Na base WEB OF SCIENCE apresentou-se de forma semelhante, trazendo estudos acerca da atuação da enfermagem e a educação permanente dos enfermeiros. $\mathrm{Na}$ base LILACS foram encontradas publicações com o predomínio da temática das lesões de pele provenientes da radioterapia.

Ao avaliar o nível de evidência dos artigos foi empregada a categorização da Agency for Healthcare Research and Quality (AHRQ). ${ }^{35}$ A qualidade das evidências é classificada em seis níveis: nível 1) metanálise de múltiplos estudos controlados; nível 2) estudo individual com delineamento experimental; nível 3) estudo com delineamento quase-experimental como estudo, sem randomização com grupo único pré e pós-teste, séries temporais ou caso-controle; nível 4) estudo com delineamento não experimental, como pesquisa descritiva correlacional e qualitativa ou estudos de caso; nível 5) relatório de casos ou dados obtidos de forma sistemática, de qualidade verificável ou dados de avaliação de programas; nível 6) opinião de autoridades respeitáveis baseada na competência clínica ou opinião de comitês de especialistas, incluindo interpretações de informações não baseadas em pesquisas.

Entre os artigos selecionados para o estudo, dois apresentaram o nível 1 de classificação, um classificou-se no nível 3, sete no nível 4 e cinco no nível 5 da AHRQ.

A seguir são apresentadas as principais temáticas desenvolvidas nos estudos incluídos, relacionados à atuação da enfermagem no serviço de radioterapia.

\section{Trabalho da Enfermagem}

O trabalho da enfermagem no serviço de radioterapia, sustentado pela Resolução $\mathrm{N}^{\circ} 358$ de $2009^{14}$ do COFEN, que dispõe sobre a SAE e o PE foi identificado em dois estudos. Baseia-se em uma problematização da realidade, onde o enfermeiro planeja a assistência de enfermagem conforme as etapas do PE: histórico, diagnóstico, planejamento, implementação e avaliação de enfermagem, para o desenvolvimento de uma assistência ética, técnica, legal, qualificada e segura. ${ }^{15,23}$ 
A dimensão da atuação da enfermagem sob uma concepção multidisciplinar foi apontada em um estudo, reportando-se essencialmente a uma visão integral da prática assistencial desenvolvida à pessoa submetida à radioterapia. ${ }^{23}$

O referencial de gestão do cuidado de enfermagem sob uma perspectiva mais ampla, incluindo, além da viabilização do $\mathrm{PE}$, os recursos humanos e instrumentais e fazendo referência à SAE, foi assinalado em um estudo. ${ }^{15}$

No tocante à consulta de enfermagem, desenvolvida na prática do enfermeiro para levantamento das necessidades individuais da pessoa submetida à radioterapia e sua família, um estudo destacou esta temática. ${ }^{15,23}$

\section{Educação Permanente em Saúde e Educação em Saúde}

Para realização de uma assistência de enfermagem de excelência, segura e qualificada nos serviços de radioterapia, a temática da Educação Permanente em Saúde (EPS) teve destaque em um estudo. Este campo de atuação, onde a radiação ionizante está presente, o estudo identificou fragilidade no que se refere às estratégias de construção de manuais de boas práticas, levando em consideração a saúde dos profissionais envolvidos neste contexto. ${ }^{26}$

\section{Humanização da Assistência}

O planejamento e execução de uma prática assistencial humanizada, percebendo os prejuízos emocionais da pessoa com câncer e seus familiares foi apontada em três estudos, destacando para a necessidade da equipe de enfermagem perceber o ser humano enquanto ser social inserido em uma família e que se apresenta fragilizado, seus medos relacionados à doença, tratamento e morte e sua vida sexual. $^{23,25,31}$

\section{Efeitos adversos da radioterapia}

As manifestações de pele e mucosas (interna e externa) são comuns em pessoas submetidas a radioterapia. Deste modo, seis estudos apontaram para a relevância da atuação da equipe de enfermagem nos aspectos relacionados à prevenção, diagnóstico precoce, tratamento e orientações ao paciente e seus familiares. Os estudos também destacaram a necessidade de um cuidado planejado, seguro, baseado em evidências, além de ações educativas em saúde. ${ }^{15,27-28,34,36}$

\section{DISCUSSÃO}

As produções científicas nacionais sobre a atuação da enfermagem nos serviços de radioterapia se intensificaram a partir de 1998, o que coincide com a publicação da Resolução No 211/1998 ${ }^{12}$ do COFEN, 
que dispõe sobre a atuação dos profissionais de enfermagem que trabalham com radiação ionizante. No que se refere ao cenário internacional, o recorte histórico escolhido mostra maior produção de estudos sobre a assistência de enfermagem ao paciente submetido à radioterapia e, também, para o estabelecimento do cuidado centrado na pessoa (person-centred care). Vale apontar que esta abordagem tem recebido crescente atenção da enfermagem ${ }^{37-39}$, considerando o paciente um participante ativo nos cuidados, tendo sua individualidade reconhecida e engajando-se numa relação terapêutica para conhecer suas necessidades pessoais de cuidado. ${ }^{40}$ Dado o profundo e extenso impacto da radioterapia em muitos aspectos do bem-estar de uma pessoa, os princípios de tal abordagem parecem idealmente adequados para o cenário de um paciente em tratamento do câncer.

O trabalho dos enfermeiros discutido nos estudos nacionais mostra que a Resolução $\mathrm{N}^{\mathrm{o}} 358$ de 15 de outubro de $2009^{14}$ do COFEN constitui um elemento fortalecedor da prática assistencial da profissão, pois ampara ética, técnica e legalmente sua assistência por meio da SAE e do PE. Nesta dimensão, a SAE é apontada como uma metodologia direcionada à gestão global da assistência, incluindo método, pessoal e instrumental, para que ocorra a operacionalização o PE, que por sua vez, é representado como método científico para o planejamento da assistência e documentação dos registros de enfermagem. ${ }^{14}$

Os estudos nacionais que abordaram a SAE e o PE apontaram a consulta de enfermagem como ferramenta crucial para o planejamento da assistência, levando em consideração que ela se utiliza das etapas do método científico para efetivar a individualização do cuidado. A consulta de enfermagem é descrita como atividade exclusiva do enfermeiro para implementar intervenções que visem à promoção, prevenção e recuperação da pessoa e da coletividade humana, estabelecendo assim, uma perspectiva multidimensional do cuidado. ${ }^{15,41}$

O PE está dividido em cinco etapas: histórico de enfermagem (processo sistemático para coleta de dados objetivos e subjetivos); diagnóstico de enfermagem (interpretação dos dados coletados para compreender a resposta da pessoa, família ou coletividade humana no processo de saúde/doença); planejamento de enfermagem (prescrição de intervenções e definição dos resultados esperados); implementação (realização das intervenções); e avaliação (processo contínuo para verificar mudanças nas respostas). ${ }^{14}$

De acordo com os estudos que apontaram o desenvolvimento do PE ao paciente submetido à radiação ionizante, foi destacada a necessidade de uma percepção integral do enfermeiro sobre o contexto no qual o paciente e família se encontram. Esta compreensão está relacionada às necessidades biopsicossociais e espirituais das pessoas envolvidas neste processo, não se excluindo nenhum aspecto destas dimensões. Neste sentido, a prática assistencial da enfermagem deve transcender as ações centradas apenas na realização de técnicas e procedimentos. ${ }^{23}$ 
Referente aos efeitos adversos da radioterapia houve ênfase em estudos nacionais sobre aspectos relacionados às manifestações de pele e mucosa provocados pela radiação ionizante, sendo as mais comuns, a radiodermite e a mucosite. Todavia, a xerostomia e a alopecia também podem estar presentes. ${ }^{15}$ A mucosite é processo de morte das células epiteliais, sem sua respectiva substituição, favorece um quadro clínico evolutivo de ulceração. Esta condição causa dificuldade para mastigar e disfagia, sendo considerada a reação aguda mais debilitante das pessoas acometidas por câncer. ${ }^{42}$

O avanço tecnológico em radioterapia aumenta a precisão no tratamento, além de reduzir os efeitos colaterais. ${ }^{43}$ Acredita-se que os efeitos adversos desse tratamento apareçam mais em publicações nacionais, em virtude da tecnologia utilizada no Brasil ainda estar aquém da utilizada em países desenvolvidos.

O planejamento da assistência do enfermeiro à pessoa submetida à radioterapia, inclui ações sistematizadas de orientações ao paciente sobre os procedimentos realizados, preparo da área disposta à radiação, posicionamento correto do paciente, exame físico, investigação da pele e mucosas, avaliação dos riscos e grau das afecções, prescrição de intervenções baseada em evidências para cobertura das lesões e as ações educativas em saúde. ${ }^{27-28,34}$

Sob a ótica de um cuidado humanizado, os estudos nesta temática evidenciaram que processo de trabalho da enfermagem ao paciente oncológico considera a atuação multiprofissional, onde se percebe o ser humano nos contextos biológicos, psicológicos, sociais e espirituais. Assim, a prática profissional deve ampliar as condutas centradas apenas na realização de técnicas e procedimentos. ${ }^{23}$

A relevância de uma assistência humanizada representa o emprego de ações que proporcionem ambientes terapêuticos para escuta qualificada, verbalização e valorização de sentimentos, identificação de problemas relacionados ao tratamento e auxílio para resolução, encaminhamentos e orientações para autocuidado, compreensão das necessidades e empatia. ${ }^{23}$ Neste entendimento, a equipe de enfermagem também deve estar atenta às percepções do paciente sobre o medo da doença, tratamento e morte, as preocupações inerentes ao seu contexto sociofamiliar, os sentimentos de impotência e o surgimento de sinais e sintomas de depressão. ${ }^{25}$

Um estudo destacou a relevância da formação e qualificação profissional frente as tecnologias emissoras de radiação ionizante, considerando que este campo está em constante evolução, em decorrência do aumento de doenças oncológicas no cenário mundial. Foram discutidas a implementação da Educação Permanente em Saúde (EPS) e educação continuada como indicadores para potencializar e qualificar o processo de trabalho da enfermagem nos serviços de radioterapia. As estratégias de EPS possibilitam ao profissional, refletir e problematizar a sua prática, fomentando assim, discussões sobre a transformação dos ambientes de trabalho. Além disso, foi mencionado pelo estudo que a proteção 
radiológica dos profissionais durante seu processo de trabalho, constitui uma importante ferramenta para a promoção da saúde, além de otimizar sua atuação na área. ${ }^{26}$

Observou-se que os estudos analisados sobre a temática processo de trabalho da enfermagem no serviço de radioterapia, apresentam suas bases apoiadas em preceitos éticos, técnicos, legais, científicos e humanizados bem fundamentados. Dessa forma, o alicerce constituído para firmar o processo de trabalho em enfermagem neste campo de atuação, representa um meio relevante para estabelecer a profissão em um patamar científico.

Para fomentar discussões sobre a relação entre a teoria e prática da atuação da enfermagem nos serviços de radioterapia, é necessário investir em estudos que fundamentem ainda melhor essa temática. Considerando a crescente evolução dos números de câncer no cenário mundial, é possível que mais serviços sejam implantados e para isso, a enfermagem necessita estar articulada à integralidade de um cuidado científico e humanizado.

No que tange às limitações do estudo, deve-se considerar que o escopo de análise desta revisão integrativa foi construído majoritariamente por artigos acerca do processo de trabalho da enfermagem. Entretanto, os relatos selecionados apresentam estratégias articuladas sobre a assistência de enfermagem no serviço de radioterapia, o que demonstra o comprometimento dos profissionais com o planejamento de uma assistência integral ao ser humano.

\section{CONSIDERAÇÕES FINAIS}

Este estudo apresentou uma revisão integrativa de literatura sobre a atuação da enfermagem no serviço de radioterapia, no recorte histórico de 1998 a 2018. Constatou-se predominância da SAE, PE e consulta de enfermagem enquanto tecnologias assistenciais relevantes para o processo de trabalho da enfermagem, além de cuidados específicos relacionados às manifestações de pele decorrentes da radioterapia e educação em saúde.

Também houve destaque para a percepção das necessidades biopsicossociais do paciente e seus familiares. Sobre as estratégias de EPS, elas podem se integrar ao processo de trabalho da profissão, promovendo intervenções acerca da formação profissional e cuidados com a saúde do trabalhador. Portanto, pode-se dizer que a atuação dos profissionais de enfermagem no serviço de radioterapia se articula à prevenção, promoção e tratamento da pessoa exposta terapeuticamente à radiação ionizante. Desta forma, o estudo fornece subsídios para uma reflexão e reconhecimento do trabalho da enfermagem neste campo de atuação, à medida que indica as principais ferramentas exploradas por estes profissionais.

Mediante o aumento expressivo de novos casos de câncer no cenário mundial, destaca-se a necessidade de mais estudos sobre esta temática, subsidiando uma assistência cada vez mais integrada e bem fundamentada. Este estudo também serve como fonte de estímulo para que outros pesquisadores 
fomentem pesquisas relacionadas ao processo de trabalho na radioterapia, contribuindo com as lacunas existentes.

\section{REFERÊNCIAS}

1. Araújo CRG, Rosas AMMTF, Menezes HF, Pinto ACS, Rodrigues BMRD. The phenomenon lived by women in nursing consultation in the gynecological brachytherapy. Texto Contexto Enferm, 2017; 26(2):e00140016. Disponível em: http://www.scielo.br/scielo.php?script=sci_arttext\&pid=S0104-07072017000200335 doi: http://dx.doi.org/10.1590/0104-07072017000140016. Acesso em: 20 mai. 2018

2. Freire MEM, Costa SFG, Lima RAG, Sawada, NO. Health-related quality of life of patients with cancer in palliative care. Texto Contexto Enferm. 27(2). 2018. Disponível em: http://www.scielo.br/scielo.php?pid=S0104-07072018000200318\&script=sci_arttext\&tlng=en. Doi: http://dx.doi.org/10.1590/0104-070720180005420016 Acesso em: 11 jun. 2018

3. Lorencetti A, Simonetti JP. As estratégias de enfrentamento de pacientes durante o tratamento de radioterapia. Rev Latinoam Enferm. 2005;13:944-50. Disponível em: http://www.scielo.br/scielo.php?script=sci_arttext\&pid=S0104-

11692005000600005\&lng=pt\&tlng=pt. $\quad$ Doi: $\quad$ http://dx.doi.org/10.1590/S010411692005000600005. Acesso em: 21 mai. 2018

4. Lee RC, Ekaette E, Kelly KL, Craighead P, Newcomb C, Dunscombe P. Implications of cancer staging uncertainties in radiation therapy decisions. Med Decis Making. 2006 May-Jun;26(3):22638. Disponível em: https://www.ncbi.nlm.nih.gov/pubmed/16751321 doi: http://dx.doi.org/10.1177/0272989X06288684. Acesso em: 22 mai. 2018

5. Donaldson L. 2006 Annual Report of the Chief Medical Officer on the State of Public Health. London: The Department of Health, 2007

6. Marks L, Light KL, Hubbs JL, Georgas DL, Jones EL, Wright MC, et al. The impact of advanced technologies on treatment deviations in radiation treatment delivery. Int J Radiat Oncol Biol Phys. 2007; 69(5):1579-1586. Disponível em: https://www.sciencedirect.com/science/article/pii/S0360301607039028?via\%3Dihub doi: https://doi.org/10.1016/j.ijrobp.2007.08.017 Acesso em: 22 mai. 2018

7. Mazur LM, Mosaly PR, Jackson M, Chang SX, Burkhardt KD, Adams RD, et al. Quantitative Assessment of Workload and Stressors in Clinical Radiation Oncology. Int J Radiat Oncol Biol Phys. 2012. Disponível em: https://www.sciencedirect.com/science/article/pii/S0360301612001368?via\%3Dihub doi: https://doi.org/10.1016/j.ijrobp.2012.01.063. Acesso em: 22 mai. 2018

8. Rose, PM. Individualized Care in Radiation Oncology. Cancer Nurs. 2016 Sep-Oct;39(5):411-22. Disponível em: https://insights.ovid.com/article/00002820-201609000-00010 doi: https://doi.org/10.1097/NCC.0000000000000332. Acesso em: 22 mai. 2018

9. BRASIL. Ministério da Saúde. Instituto Nacional do Câncer. Ações de Enfermagem para o controle do câncer: Uma proposta de integração ensino-serviço. Rio de Janeiro: INCA; 2008. Acesso em: 22 mai. 2018

10. Cacelli EMN, Pereira MLM, Rapoport A. Avaliação da mucosite e xerostomia como complicações do tratamento de radioterapia no câncer de boca e orofaringe. Rev Bras Cir Cabeça Pescoço. 2009;38:80-3. Disponível: https://www.sbccp.org.br/wp-content/uploads/2014/11/art_4.pdf.

Acesso em: 22 mai. 2018 
11. INCA. Expansão da Assistência Oncológia (Projeto EXPANDE) 2018. Disponível em: http://www2.inca.gov.br/wps/wcm/connect/acoes_programas/site/home/nobrasil/projeto_expande Acesso em: 22 mai. 2018

12. COFEN. Conselho Federal de Enfermagem. Resolução no 211/1998. Dispõe sobre a atuação dos profissionais de enfermagem que trabalham com radiação ionizante. In: Portal do 7 - COFEN. Disponível em: http://www.cofen.gov.br/resoluo-cofen-2111998_4258.html>. Acesso em: 23 mai. 2018.

13. COFEN. Brasília (DF): COFEN; 2011. Disponível em: http://www.portalcofen.gov.br/sitenovo/sites/defau lt/files/esquisaprofissionais.pdf. acesso em: 10 jun. 2018.

14. Brasil. Conselho Federal de Enfermagem. Resolução N ${ }^{\circ}$. 358/2009. In: Portal do COFEN. Disponível em: <http://www.cofen.gov.br/resoluo-cofen-3582009_4384.html>. Acesso em: 11 jun. de 2018.

15. Souza NR, Santos ICRV, Bushatsky M, Figueiredo EG, Melo JTS, Santos CS. Atuação de enfermeiros em serviços de radioterapia. Rev enferm UERJ, Rio de Janeiro, 2017; 25:e26130. Disponível em: http://www.e-publicacoes.uerj.br/index.php/enfermagemuerj/article/view/26130 Acesso em: 11 jun. 2018

16. Araújo CRG, Rosas AMMTF. A consulta de enfermagem para clientes e seus cuidadores no setor de radioterapia de hospital universitário. Rev Enferm UERJ. 2008;16:364-9. Disponível em: http://www.facenf.uerj.br/v16n3/v16n3a11.pdf Acesso em: 11 jun. 2018

17. Kelvin JF, Moore-Higgs GJ, Maher KE, Dubey AK, Austin-Seymour MM, Daly NR, et al. Nonphysician practitioners in radiation oncology: advanced practice nurses and physician assistants. Int J Radiat Oncol Biol Phys. 1999 Sep 1;45(2):255-63. Disponível em: https://doi.org/10.1016/S0360-3016(99)00180-7. Acesso em: 11 jun. 2018

18. Whittemore R, Knafl K. The integrative review: updated methodology. J Adv Nurs. 2005 Dec; 52(5):546-53. Disponível em: https://onlinelibrary.wiley.com/doi/full/10.1111/j.13652648.2005.03621.x doi: https://doi.org/10.1111/j.1365-2648.2005.03621.x Acesso em: 09 jun. 2018

19. Mendes KS, Silveira RCCP, Galvão CM. Integrative literature review: A research method to incorporate evidence in health care and nursing. Texto Contexto Enferm, 17(4):758-64, out./dez. 2008. Disponível em: http://www.scielo.br/scielo.php?script=sci_arttext\&pid=S010407072008000400018 doi: http://dx.doi.org/10.1590/S0104-07072008000400018 Acesso em: 09 jun. 2018

20. Ganong LH. Integrative Reviews of Nursing. Rev Nurs Health. 1987;10(1):1-11. Acesso em: 28 dez. 2018.

21. BRASIL. RESOLUÇÃO COFEN-211/1998 Dispõe sobre a atuação dos profissionais de Enfermagem que trabalham com radiação ionizante. 1998. Acesso em: 28 dez. 2018.

22. Faithfull S, Corner J, Meyer L, Huddart R, Dearnaley D. Evaluation of nurse-led follow up for patients undergoing pelvic radiotherapy. British Journal of Cancer. 2001 85(12):1853-64. Acesso em: 11 jun 2018. Disponível em:https://www.nature.com/articles/6692173.pdf?origin=ppub Acesso em: 28 dez. 2018.

23. Costa CA, Lunardi WDF, Soares NV. Humanized assistance with an oncology patient: reflections with the health team. Rev. bras. enferm. [online]. 2003, 56(3):310-314. Disponível em: ISSN 0034-7167. http://dx.doi.org/10.1590/S0034-71672003000300019. Acesso em: 11 jun. 2018.

24. Cruickshank S, Kennedy C, Lockhart K, Dosser I, Dallas L. Specialist breast care nurses for supportive care of women with breast cancer. Cochrane Breast Cancer Group. Disponível em: < 10.1002/14651858.CD005634.pub2> Acesso em: 28 dez. 2018.

25. Muniz RF, Zago MMF, Schwartz E. As teias da sobrevivência oncológica: com a vida de novo. Texto Contexto Enferm, 2009 Jan-Mar; 18(1): 25-32. Disponível em: 
http://www.scielo.br/scielo.php?pid=S0104-07072009000100003\&script=sci_abstract\&tlng=pt.

Doi: http://dx.doi.org/10.1590/S0104-07072009000100003. Acesso em: 28 dez. 2018.

26. Flôr RC, Gelbcke FL. Tecnologias emissoras de radiação ionizante e a necessidade de educação permanente para uma práxis segura da enfermagem radiológica. Rev Bras Enferm, Brasília 2009 set-out; 62(5): 766-70. Disponível em: http://www.scielo.br/scielo.php?script=sci_arttext\&pid=S0034-71672009000500021 Acesso em: 28 dez. 2018.

27. Gondim FM, Gomes IP, Firmino F. Prevenção e tratamento da mucosite oral. Rev. enferm. UERJ, Rio de Janeiro, 2010 jan/mar; 18(1):67-74. Disponível em: <http://www.facenf.uerj.br/v18n1/v18n1a12.pdf> Acesso em: 28 dez. 2018.

28. Santos RCS, Dias RS, Giordani AJ, Segreto RA, Segreto HRC. Mucosite em pacientes portadores de câncer de cabeça e pescoço submetidos à radioquimioterapia. Rev Esc Enferm USP 2011; 45(6):1338-44. Disponível em: http://www.scielo.br/scielo.php?script=sci_arttext\&pid=S008062342011000600009 Acesso em: 28 dez. 2018.

29. Waal I, Van D, et al. Early diagnosis in primary oral cancer: is it possible?. Medicina Oral Patología Oral y Cirugia Bucal, [s.1.]. 2011;16(3):300-305. Disponível em: https://www.ncbi.nlm.nih.gov/pubmed/21441877. Doi: http://dx.doi.org/10.4317/medoral.16.e300. Acesso em: 28 dez. 2018.

30. Rose P. Person centred nursing care in radiation oncology: A case study. European Journal of Oncology Nursing .17(5):554-62. October 2013. Disponível em: https://doi.org/10.1016/j.ejon.2013.02.001 Acesso em: 28 dez. 2018.

31. Alcantara LS, Oliveira ACM, Guedes MTS, Santos MCM, Diniz DR, Soares E. Interdisciplinaridade e Integralidade: a Abordagem do Assistente Social e do Enfermeiro no INCA. Rev Bras Cancerol. 2014; 60(2):109-118. Disponível em: https://rbc.inca.gov.br/index2.php. Acesso em: 28 dez. 2018.

32. Lotfi-Jam K, Schofield P, Aranda S. Profile and predictors of global distress: Can the DT guide nursing practice in prostate cancer? 12(1):5-14. 2014. Disponível em: < https://doi.org/10.1017/S1478951513000060> Acesso em: 28 dez. 2018.

33. Schofield P, Gough K, Lotfi-Jam K, Bergin R, Ugalde A, Dudgeon P, et al. Nurse-led group consultation intervention reduces depressive symptoms in men with localised prostate cancer: a cluster randomised controlled trial. BMC Cancer, 2016. Disponível em: < https://doi.org/10.1186/s12885-016-2687-1>. Acesso em: 02 jan. 2019.

34. Tsuchihashi Y, Matsunari Y, Kanamaru Y. Survey of difficult experiences of nurses caring for patients undergoing radiation therapy: An analysis of factors in difficult cases. Asia-pacific Journal Of Oncology Nursing, 2017. Medknow. Disponível em: http://dx.doi.org/10.4103/apjon.apjon_48_17 Acesso em: 03 jan. 2019.

35. Agency for HealthCare Research and Quality. Rockville, MD. [Cited 2019 Ago 14]. Available from: http://www.qualityindicators.ahrq.gov. Acesso em: 05 jan. 2019.

36. Sanchez SE, Céron MVJ, Vela RS, Munoz GMJ. Nursing care protocol on management of radiodermitis. Revista Enfermagem UERJ. 2016 Jan; 39 (1): 38-47. Acesso em: 102 fev. 2019.

37. Nandini V, Sridhar C.N, Usharani M.R, Kumar J.P, Saleen N. 2011. Incorporating person centred care principles into an ongoing comprehensive cancer management program: an experiential account. Indian Journal of Palliative Care 17 (Suppl), S61eS67. Acesso em: 16 fev. 2019.

38. Price B., 2006. Exploring person-centred care. Nursing Standard 20(50):49-56. Acesso em: 17 fev. 2019.

39. Coulter A, Ellins J. 2006. Patient-focused Interventions: a Review of the Literature. The Health Foundation and The Picker Institute Europe, London. Acesso em: 18 fev. 2019.

40. Slater P. 2006. Person-centredness: a concept analysis. Contemporary Nurse 23 (1), 135. Acesso em: 18 fev. 2019. 
41. BRASIL. Resolução COFEN No 0544/2017. Revoga a Resolução Cofen no 159/1993 - Consulta de Enfermagem. Disponível em: http://www.cofen.gov.br/resolucao-cofen-no05442017_52029.html. Acesso em: 19 fev. 2019.

42. Naidu MUR, Ramana GV, Rani PU, Mohan IK, Suman A, Roy P. Chemotherapy induced and/or therapy-induced oral mucositis-complicating the treatment of cancer. Neoplasia. 2004;6(5):42331. Acesso em: 19 fev. 2019.

43. Haughton ME, Chan MD, Watabe K, Bonomi M, Debinski W, Lesser GJ, Ruiz J.Treatment of brain metastases of lung cancer in the era of precision medicine. Front Biosci (Elite Ed). 2016 Jan 1;8:219-32. Disponível em: https://www.bioscience.org/2016/v8e/af/763/fulltext.htm. Acesso em: 19 fev. 2019. 